\title{
BMJ Open Tai Chi for stroke rehabilitation: protocol for a systematic review
}

\author{
Yong Zhang, ${ }^{1,2,3,4}$ Shanshan Wang, ${ }^{2,4}$ Pei Chen, ${ }^{3}$ Xiaoshu Zhu, ${ }^{2}$ Zongheng Li ${ }^{1}$
}

To cite: Zhang Y, Wang S, Chen $\mathrm{P}$, et al. Tai Chi for stroke rehabilitation: protocol for a systematic review. BMJ Open 2016:6:e010866. doi:10.1136/bmjopen-2015010866

- Prepublication history for this paper is available online. To view these files please visit the journal online (http://dx.doi.org/10.1136/ bmjopen-2015-010866).

Received 14 December 2015 Revised 7 May 2016 Accepted 23 May 2016
CrossMark

For numbered affiliations see end of article.

Correspondence to Dr Zongheng Li; lee_zongheng@163.com

\section{ABSTRACT}

Introduction: Stroke is a major cause of death and disability, and imposes a huge burden and significant workload for patients, their families and society. As a special form of physical activity, Tai Chi is may be useful for stroke rehabilitation. The objective of this review is to systematically evaluate the efficacy and safety of Tai Chi for rehabilitation in stroke patients.

Methods and analysis: We will conduct a systematic search of the following electronic databases from their inception to 31 October 2015: MEDLINE, EMBASE, the Cochrane Library, the Chinese BioMedical Literature Database (CBM), the Chinese National Knowledge Infrastructure (CNKI), the Chinese Science and Technology Periodical Database (VIP), Wanfang and the Chinese Dissertation Database. All relevant randomised controlled trials (RCTs) in English and Chinese will be included. The main outcomes will be changes in the neurological function of patients and in independence in activities of daily living. Adverse events, adherence, costs and the cost effectiveness of Tai Chi will also be assessed. Two independent reviewers will select studies, extract data and assess quality. Review Manager 5.3 will be used for assessment of risk of bias, data synthesis and subgroup analysis.

Ethics and dissemination: This systematic review does not require formal ethical approval because all data will be analysed anonymously. Results will provide a general overview and evidence concerning the efficacy and safety of Tai Chi for stroke rehabilitation. Findings will be disseminated through peer-reviewed publications.

Trial registration number: CRD42015026999.

\section{INTRODUCTION}

\section{Description of the condition}

Stroke is the second most common cause of death and a major cause of disability worldwide. ${ }^{1}$ It greatly affects the quality of life of survivors and imposes a huge public health burden. $^{2}$ As the population ages and lifestyles change, this burden is projected to increase markedly over the next 20 years, especially in developing countries. ${ }^{3}$ A recent study indicated that stroke mortality rates have decreased worldwide but that the

\section{Strengths and limitations of this study}

- The current systematic review will reassess the efficacy and safety of Tai Chi for stroke rehabilitation and will provide further clinical evidence for both clinicians and patients.

- There may be language bias as only studies published in English and Chinese will be included, so relevant studies in other languages may be missed.

- There may be significant heterogeneity due to the different types of Tai Chi and duration and frequency of practice.

absolute number of new stroke patients, stroke survivors with functional disability, stroke-related deaths, and the overall global burden of stroke have increased in the past two decades. ${ }^{2}$ An epidemiological study showed that there were over 7 million stroke survivors in China, approximately $70 \%$ of whom had functional disability. ${ }^{4}$ Stroke is the leading cause of disability and impacts greatly on patients' quality of life, resulting in a huge burden and significant workload for families and societies. ${ }^{5} 6$ This situation demands effective modalities for stroke rehabilitation.

\section{Description of the intervention}

Tai Chi (also known as Tai Ji or Tai Chi Chuan) is a form of physical activity that has been widely practiced in China and elsewhere. Tai Chi originated in China as a martial art hundreds of years ago. Combining physical movement, meditation and controlled breathing to achieve a mindbody connection, Tai Chi induces relaxation and mental tranquillity and improves balance, postural control, movement coordination, strength and flexibility. ${ }^{7-9}$ It has become a popular exercise worldwide in recent years. ${ }^{10}$ Numerous studies have been conducted on the clinical application of Tai Chi and have validated its effects in improving various symptoms. ${ }^{10}$ Previous studies indicated that Tai Chi can be prescribed as a beneficial and safe exercise for neurological 
disease, ${ }^{11}{ }^{12}$ cardiovascular disease, ${ }^{13}{ }^{14}$ orthopaedic disease, ${ }^{9} \quad 15$ rheumatological disease, ${ }^{16}$ cancer ${ }^{17}$ and some other conditions.

Tai Chi has also been incorporated into stroke rehabilitation programmes. ${ }^{18} 19$ Previous studies and reviews suggested that Tai Chi is safe and feasible for stroke patients with functional disability and may serve as an additional exercise modality in stroke rehabilitation. It has been demonstrated that Tai Chi is beneficial in improving motor function, ${ }^{20}$ standing balance ${ }^{21} 22$ and quality of life, ${ }^{20}{ }^{21}$ and in reducing fall rates $^{20}$ in stroke patients. In addition, some ongoing studies are investigating the effects of Tai Chi for stroke rehabilitation. ${ }^{23}{ }^{24}$ Thus, it is necessary to systematically review the efficacy and safety of Tai Chi for stroke rehabilitation and provide further clinical evidence for the benefit of both clinicians and patients.

\section{How the intervention might work}

Tai Chi may be helpful for stroke patients as its practice involves mechanisms similar those of conventional stroke rehabilitation techniques, like the Bobath programme and proprioceptive neuromuscular facilitation. ${ }^{18}$ Most Tai Chi movements are helical and aimed at strengthening the limbs and core muscles of the abdomen and back. ${ }^{25}$ This correlates with conventional stroke rehabilitation techniques which also focus on strength exercises for the limbs and the trunk. Since Tai Chi is practiced mostly on one foot, it is also a weightbearing exercise that improves balance similar to conventional rehabilitation exercises. ${ }^{19}$ The requirement for deep breathing and relaxation of the body and mind when practicing Tai Chi is consistent with stroke rehabilitation therapies that encourage patients to relax and stay calm to achieve better recovery. One of the most important principles of Tai Chi is 'conquering the unyielding with the yielding', which correlates with physiotherapy in treating spasticity with gentle manipulation.

\section{OBJECTIVES}

The objective of this review is to systematically evaluate the efficacy and safety of Tai Chi for rehabilitation in stroke patients.

\section{METHODS AND ANALYSIS}

Types of studies

We will only include clinical randomised controlled trials (RCTs) in English and Chinese without any restrictions on publication status. Non-RCTs, quasi-RCTs and uncontrolled clinical trials such as case studies will be excluded.

\section{Types of participants}

Trials involving patients of any age with ischaemic or haemorrhagic stroke will be included. Stroke must be diagnosed according to the WHO definition (rapidly developing clinical signs of focal (or global) disturbance of cerebral function, lasting more than 24 hours or leading to death, with no apparent cause other than that of vascular origin) ${ }^{26}$ or confirmed by CT or MRI. There will be no limitation in relation to time from the onset of stroke. Patients with subarachnoid haemorrhage or subdural haematoma will be excluded.

\section{Types of interventions}

Similarly to other mind-body interventions like yoga and Qigong, Tai Chi is also inherently varied and heterogeneous which makes standardisation difficult. Thus, we will accept all types of Tai Chi interventions regardless of their form, style, duration and frequency. However, stratified analysis according to these variables will be performed if sufficient studies are included. The intervention in the treatment group should be Tai Chi exercises with or without conventional rehabilitative treatment. The control intervention will include conventional rehabilitative treatment, other forms of exercises, or no treatment. Some other co-interventions, such as basic medication for stroke, lifestyle modifications for stroke, stroke diets and stroke education programmes, are acceptable on condition that the same co-interventions are simultaneously applied in all arms of a study.

\section{Types of outcome assessments \\ Primary outcomes}

The primary outcomes of this review will focus on changes in patients' neurological function, balance and independence in activities of daily living. This will encompass assessment tools based on the National Institutes of Health Stroke Scale (NIHSS), Fugl-Meyer Assessment (FMA), Berg Balance Scale (BBS), modified Rankin Scale (mRS), Barthel Index (BI), Stroke Specific Quality of Life Scale (SSQOL) or the researchers' own definition.

\section{Secondary outcomes}

Secondary outcome measures will include possible mental health improvements related to the practice of Tai Chi, adverse events associated with Tai Chi, all-cause death during the entire treatment and follow-up period, adherence to Tai Chi, and the costs and cost effectiveness of Tai Chi.

\section{Search methods for identification of studies Electronic searches}

We will conduct a systematic search of the following electronic databases from inception to 31 October 2015: MEDLINE, EMBASE, the Cochrane Library, the Chinese BioMedical Literature Database (CBM), the Chinese National Knowledge Infrastructure (CNKI), the Chinese Science and Technology Periodical Database (VIP), Wanfang and the Chinese Dissertation Database (CDD).

We have developed the MEDLINE search strategy (see table 1) based on guidance from the Cochrane handbook and will apply similar strategies for other electronic databases. 


\begin{tabular}{|c|c|}
\hline Number & Search items \\
\hline 1 & exp tai chi/ or tai ji/ \\
\hline 2 & $\begin{array}{l}\text { (tai chi or tai ji or taichi or tai chi chuan or taichi } \\
\text { chuan or taiji or tai ji quan or taiji quan or } \\
\text { martial arts or shadowbox\$).tw. }\end{array}$ \\
\hline 3 & or/ 1-2 \\
\hline 4 & $\begin{array}{l}\text { cerebrovascular disorders/ or exp basal ganglia } \\
\text { cerebrovascular disease/ or exp brain ischemia/ } \\
\text { or exp carotid artery diseases/ or exp } \\
\text { intracranial arterial diseases/ or exp "intracranial } \\
\text { embolism and thrombosis"/ or exp intracranial } \\
\text { hemorrhages/ or stroke/ or exp brain infarction/ } \\
\text { or vasospasm, intracranial/ or vertebral artery } \\
\text { dissection/ }\end{array}$ \\
\hline 5 & $\begin{array}{l}\text { (stroke or poststroke or post-stroke or } \\
\text { cerebrovasc } \$ \text { or brain vasc } \$ \text { or cerebral vasc\$ } \\
\text { or cva } \$ \text { or apoplex\$).tw. }\end{array}$ \\
\hline 6 & $\begin{array}{l}\text { ((brain } \$ \text { or cerebr } \$ \text { or cerebell\$ or intracran } \$ \text { or } \\
\text { intracerebral) adj5 (isch?emi } \$ \text { or infarct\$ or } \\
\text { thrombo } \$ \text { or emboli } \$ \text { or occlus } \$)) \text {.tw. }\end{array}$ \\
\hline 7 & $\begin{array}{l}\text { ((brain } \$ \text { or cerebr } \$ \text { or cerebell\$ or intracerebral } \\
\text { or intracranial or subarachnoid) adj5 } \\
\text { (haemorrhage } \$ \text { or hemorrhage } \$ \text { or } \\
\text { haematoma } \$ \text { or hematoma } \$ \text { or bleed } \$) \text { ).tw. }\end{array}$ \\
\hline 8 & hemiplegia/ or exp paresis/ \\
\hline 9 & (hemipleg\$ or hemipar\$ or paresis or paretic).tw. \\
\hline 10 & or/ 4-9 \\
\hline 11 & 3 and 10 \\
\hline
\end{tabular}

\section{Other resources}

The WHO International Clinical Trials Registry Platform (ICTRP) and its Registry Network and the reference lists of related reviews and retrieved articles will be checked for additional studies. We will also scan the abstracts of non-English language studies if they are available in English. We will also search relevant conference papers on this topic.

\section{Data collection and analysis \\ Selection of studies}

Two review authors (YZ and SW) will independently check the titles and abstracts of retrieved results and select all potentially relevant references. All records will be managed with Endnote X7 in a separate database. The two reviewers will then independently read the full texts and select studies to be included based on our predetermined inclusion criteria. In case of unclear information or missing data, we will contact the original authors for clarification. Disagreements regarding inclusion will be resolved by discussion and consultation with an experienced reviewer (XZ). Details of the entire selection procedure will be shown in a PRISMA flow chart (see figure 1).

\section{Data extraction and management}

Two review authors (YZ and SW) will independently carry out data extraction using a piloted data extraction form which will be developed by all authors with reference to previously published systematic review papers on Tai Chi and stroke. Data will include general information on the publications (reference identification, authors, country, journal name, year of publication, etc), details of study design (sample size, randomisation, blinding, etc), participants (inclusion and exclusion criteria, age, gender, disease duration, etc), interventions (types of Tai Chi, types of control, duration, frequency, etc) and outcomes (observation time points, measurement tools, follow-up, adherence, adverse events, costs and cost effectiveness, etc). The original authors will be contacted in case of missing data. Where there is disagreement regarding data extraction, a third experienced reviewer (XZ) will be consulted for consensus.

\section{Assessment of risk of bias in included studies}

Risk of bias will be assessed by two independent authors (YZ and SW) using the Cochrane risk of bias tool recommended by the Cochrane reviewer's handbook. Any disagreements will be resolved through consultation with a third experienced reviewer (XZ). The following will be assessed for risk of bias: selection, performance, detection, attrition, reporting and other factors. We will not consider bias among participants and personnel because the practice of Tai Chi makes blinding impossible. All included studies will be categorised into three categories: high, unclear and low risk of bias.

\section{Measure of treatment effect}

We will calculate risk ratios (RRs) with 95\% CIs for dichotomous outcomes and mean differences (MDs) or standard mean differences (SMDs) with 95\% CIs for continuous outcomes.

\section{Unit of analysis issues}

The primary unit of analysis will be all individuals participating in the trials. In case of three or more different intervention groups within a trial, we will present pairwise comparison results through different subgroups of interventions. If available, we will combine relevant groups to provide a single pairwise comparison with the Tai Chi group.

\section{Dealing with missing data}

We will contact the original authors in case of missing data. If this fails to elicit the required information, we will only analyse the available data and address the potential impact of these missing data on the results of the review in the discussion section.

\section{Assessment of heterogeneity}

We will check the results of the $\chi^{2}$ test (significance level: 0.1$)$ to assess the heterogeneity of included studies and the $\mathrm{I}^{2}$ statistic to quantify inconsistency. An $\mathrm{I}^{2}$ value of $50 \%$ or higher will indicate the presence of substantial heterogeneity. If there is a low level of heterogeneity among included studies, we will synthesise the results in 
Figure 1 Flow diagram of the study selection process. CBM, Chinese BioMedical Literature Database; CDD, Chinese Dissertation Database; CNKI, Chinese National Knowledge Infrastructure; RCT, randomised controlled trial; VIP, Chinese Science and Technology Periodical Database.

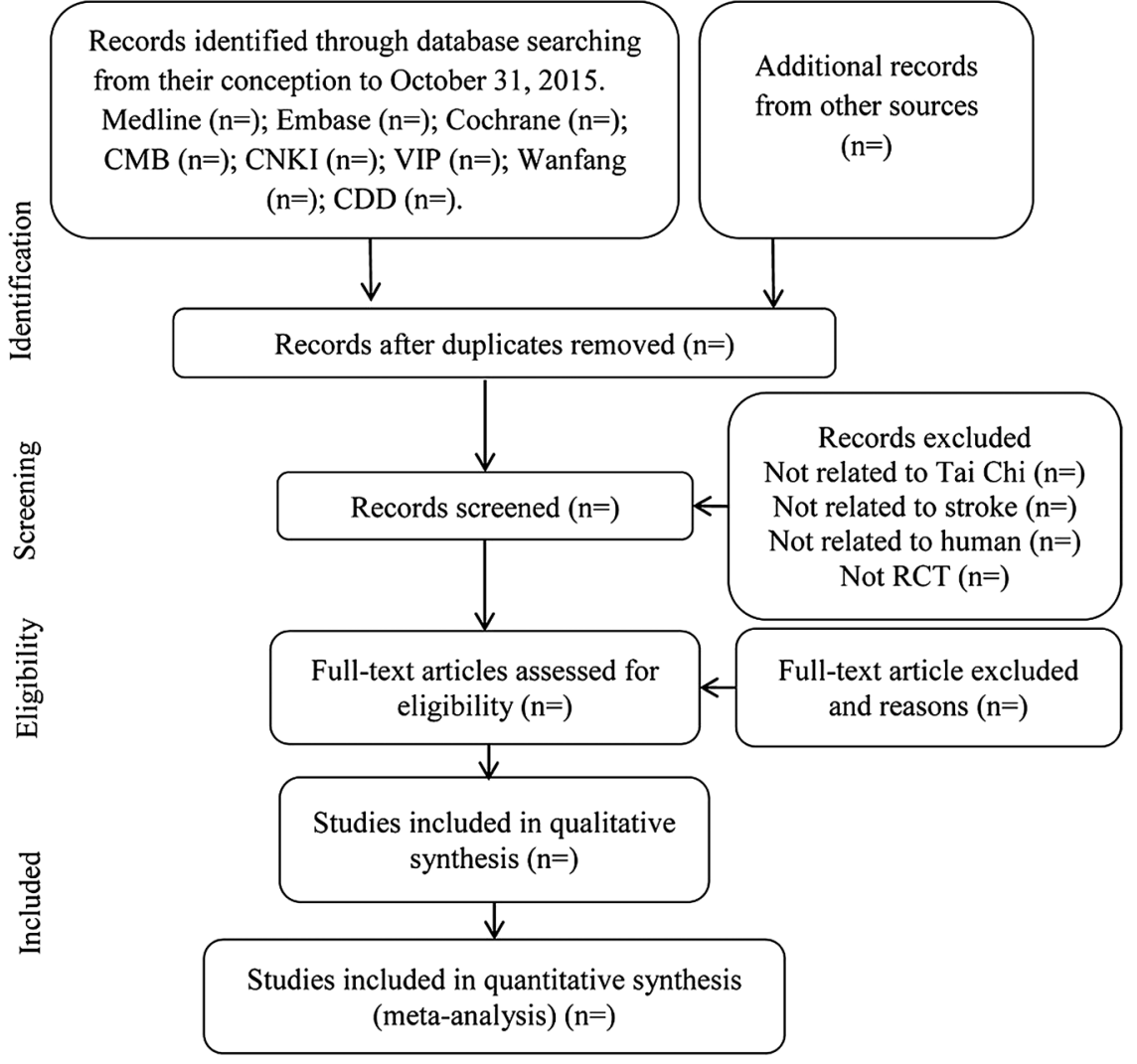

a meta-analysis. In case of substantial heterogeneity, we will perform a systematic synthesis instead.

\section{Assessment of reporting biases}

We will assess reporting bias according to the CONSORT criteria and will generate funnel plots to assess reporting bias if sufficient studies are included. We will try to explore possible interpretations other than publication bias and language bias if funnel plots are asymmetric.

\section{Data synthesis}

Data synthesis will be performed with Cochrane Review Manager (V.5.3). We will adopt the fixed effects model or random effects model depending on the results of heterogeneity assessment.

\section{Subgroup analysis}

We plan to carry out subgroup analysis if sufficient comparable studies are identified. We intend to stratify the results by duration, style and frequency of Tai Chi. We will also focus on subgroup analyses of comparison between Tai Chi and different stroke rehabilitation treatments. The incidence rates of different types of adverse events will also be calculated.

\section{Sensitivity analysis}

To ensure the robustness of evidence, we will perform sensitivity analysis to assess the impact of studies with a high risk of bias. We will compare the results to decide whether lower quality studies should be excluded on the basis of sample size, strength of evidence and influence on pooled effective size.

\section{Grading the quality of evidence}

To help health professional make decisions regarding individual patients, we will further evaluate the quality of evidence for outcomes by using the GRADE system. We will also consider the quality of evidence, potential benefits and harms, study context and patients' value when interpreting the results.

\section{Ethics and dissemination}

This systematic review does not require formal ethical approval because all data used will be anonymous with no concerns regarding privacy. Results will provide a general overview and evidence concerning the effectiveness and safety of Tai Chi for stroke rehabilitation. Findings will be disseminated through peer-reviewed publications.

\section{DISCUSSION} determine a beneficial effect of Tai Chi for stroke rehabilitation. $^{27}$ Another Cochrane review aimed at evaluating the effectiveness of Tai Chi on dependency and motor function for the recovery of stroke patients was registered in December 2012. ${ }^{28}$ However, the authors have failed to provide any results of their review.
The previous review published 3 years ago failed to 
Nearly 10 new RCTs of Tai Chi applied for stoke rehabilitation have been published in the past 3 years. Thus, it is necessary to conduct a systematic review to reassess the efficacy and safety of Tai Chi for stroke rehabilitation. In the current paper, we have described the protocol for a systematic review designed to assess the efficacy and safety of Tai Chi for stroke rehabilitation. We hope that our results may be of benefit to patients and provide further clinical evidence for both clinicians and patients making decisions regarding the practice of Tai Chi for stroke rehabilitation.

However, the proposed systematic review has some potential limitations. There may be a language bias as we will only include studies published in English and Chinese, which mean some relevant studies in other languages (eg, Korean and Japanese) may be missed. Another limitation is that there may be significant heterogeneity due to the various different types of Tai Chi, styles, duration and frequency.

\section{Author affiliations}

${ }^{1}$ Department of Rehabilitation, Dongzhimen Hospital, The First Affiliated Hospital of Beijing University of Chinese Medicine, Beijing, China ${ }^{2}$ National Institute of Complementary Medicine, Western Sydney University, Sydney, New South Wales, Australia

${ }^{3}$ Department of Neurology and Stroke Center, Dongzhimen Hospital, The First Affiliated Hospital of Beijing University of Chinese Medicine, Beijing, China ${ }^{4}$ Department of International Communications, Beijing University of Chinese Medicine, Beijing, China

Contributors $Y Z, X Z$ and ZL conceived the study. The protocol was drafted by $Y Z$, and revised by $X Z$ and $Z L$. YZ, SW, PC and $X Z$ developed the search strategy. $Y Z$ and $S W$ will independently work on data extraction and synthesis.

Funding This work is supported by the following: the Middle-aged Teachers Research Funds of Beijing University of Chinese Medicine (grant no. 2015-JYB-JSMS082), the Second Round of Special Project for Chinese Medicine Clinical Research Base of the China State Administration of Traditional Chinese Medicine (grant no. JDZX2015312) and the Oversea Teachers Program of China Confucius Institute Headquarters (Hanban).

\section{Competing interests None declared.}

Provenance and peer review Not commissioned; externally peer reviewed.

Data sharing statement The results of the review will be disseminated through peer-reviewed publications.

Open Access This is an Open Access article distributed in accordance with the Creative Commons Attribution Non Commercial (CC BY-NC 4.0) license, which permits others to distribute, remix, adapt, build upon this work noncommercially, and license their derivative works on different terms, provided the original work is properly cited and the use is non-commercial. See: http:// creativecommons.org/licenses/by-nc/4.0/

\section{REFERENCES}

1. Johnston SC, Mendis S, Mathers CD. Global variation in stroke burden and mortality: estimates from monitoring, surveillance, and modelling. Lancet Neurol 2009;8:345-54.

2. Feigin VL, Forouzanfar MH, Krishnamurthi R, et al. Global and regional burden of stroke during 1990-2010: findings from the Global Burden of Disease Study 2010. Lancet 2014;383:245-54.
3. Giroud $M$, Jacquin $A$, Bejot $Y$. The worldwide landscape of stroke in the 21st century. Lancet 2014;383:195-7.

4. Liu M, Wu B, Wang WZ, et al. Stroke in China: epidemiology, prevention, and management strategies. Lancet Neurol 2007;6:456-64.

5. Liu L, Wang D, Wong KS, et al. Stroke and stroke care in China: huge burden, significant workload, and a national priority. Stroke 2011;42:3651-4.

6. Zhang $Y$, Jin $H$, Ma D, et al. Efficacy of integrated rehabilitation techniques of Traditional Chinese Medicine for ischemic stroke: a randomized controlled trial. Am J Chin Med 2013;41:971-81.

7. Yang GY, Wang LQ, Ren J, et al. Evidence base of clinical studies on Tai Chi: a bibliometric analysis. PLoS ONE 2015;10:e0120655.

8. Zhang L, Layne C, Lowder T, et al. A review focused on the psychological effectiveness of tai chi on different populations. Evid Based Complement Alternat Med 2012;2012:e678107.

9. Wang $\mathrm{C}$, Schmid $\mathrm{CH}$, Rones $\mathrm{R}$, et al. A randomized trial of tai chi for fibromyalgia. N Engl J Med 2010;363:743-54.

10. Lan C, Chen S-Y, Lai J-S, et al. Tai Chi chuan in medicine and health promotion. Evid Based Complement Alternat Med 2013;2013: e502131.

11. Yang $Y, L i X Y$, Gong L, et al. Tai Chi for improvement of motor function, balance and gait in Parkinson's disease: a systematic review and meta-analysis. PLOS ONE 2014;9:e102942.

12. Li F, Harmer P, Fitzgerald K, et al. Tai chi and postural stability in patients with Parkinson's disease. N Engl J Med 2012;366:511-9.

13. Lee MS, Pittler MH, Taylor-Piliae RE, et al. Tai chi for cardiovascular disease and its risk factors: a systematic review. J Hypertens 2007;25:1974-5.

14. Lan C, Chen SY, Wong MK, et al. Tai chi chuan exercise for patients with cardiovascular disease. Evid Based Complement Alternat Med 2013;2013:e983208.

15. Song QH, Zhang QH, Xu RM, et al. Effect of Tai-chi exercise on lower limb muscle strength, bone mineral density and balance function of elderly women. Int J Clin Exp Med 2014;7:1569-76.

16. Lee MS, Pittler MH, Ernst E. Tai chi for rheumatoid arthritis: systematic review. Rheumatology (Oxford) 2007;46:1648-51

17. Pan $\mathrm{Y}$, Yang K, Shi $\mathrm{X}$, et al. Tai chi chuan exercise for patients with breast cancer: a systematic review and meta-analysis. Evid Based Complement Alternat Med 2015;2015:e535237.

18. Yu MH, Wang WD. Tai Chi exercise and hemiplegia rehabilitation. Chin J Rehabil Theory Pract 2002;18:447-8.

19. Taylor-Piliae RE, Haskell WL. Tai Chi exercise and stroke rehabilitation. Topic Stroke Rehabil 2007;14:9-22.

20. Taylor-Piliae RE, Hoke TM, Hepworth JT, et al. Effect of Tai Chi on physical function, fall rates and quality of life among older stroke survivors. Arch Phys Med Rehabil 2014;95:816-24.

21. Kim H, Kim YL, Lee SM. Effects of therapeutic Tai Chi on balance, gait, and quality of life in chronic stroke patients. Int $J$ Rehabil Res 2015;38:156-61.

22. Au-Yeung SS, Hui-Chan CW, Tang JC. Short-form Tai Chi improves standing balance of people with chronic stroke. Neurorehabil Neural Repair 2009;23:515-22.

23. Tao J, Rao T, Lin L, et al. Evaluation of Tai Chi Yunshou exercises on community-based stroke patients with balance dysfunction: a study protocol of a cluster randomized controlled trial. BMC Complement Altern Med 2015;15:31.

24. Zhang $\mathrm{Y}$, Liu H, Zhou L, et al. Applying Tai Chi as a rehabilitation program for stroke patients in the recovery phase: study protocol for a randomized controlled trial. Trials 2014;15:484.

25. Lan C, Lai JS, Chen SY, et al. Tai Chi Chuan to improve muscular strength and endurance in elderly individuals: a pilot study. Arch Phys Med Rehabil 2000;81:604-7.

26. World Health Organisation. Stroke-1989. Recommendations on stroke prevention, diagnosis, and therapy. Report of the WHO task force on stroke and other cerebrovascular disorders. Stroke 1989:20:1407-31.

27. Ding M. Tai Chi for stroke rehabilitation: a focused review. Am J Phys Med Rehabil 2012;91:1091-6.

28. Xu C, Zhang HW, Leung AW, et al. Tai Chi for improving recovery after stroke (protocol). Cochrane Database Syst Rev 2012;(12): CD010207. 